\title{
Proton-beam therapy or photon-beam radiosurgery for WHO grade I meningiomas?
}

\author{
Thomas Mindermann ${ }^{1}$ (D) Andreas Mack $^{2}$
}

Received: 28 September 2017 / Accepted: 2 October 2017 /Published online: 13 October 2017

(C) Springer-Verlag GmbH Austria 2017

Proton-beam therapy (PBT) has been in use since the early 1960s [1]. Typically, PBT has been applied in rare or paediatric neoplasms because of its costs, its specific physical properties and its rare availability. PBT's share of radiotherapy was $0.6 \%$ in the US in 2012 [4]. In this issue of Acta Neurochirurgica, Vlachogiannis et al. [3] present their experience with PBT in 170 patients with WHO grade I meningiomas. As opposed to the classical use of PBT, their patients have neither a rare tumour type nor do they belong to the paediatric age group. Reports on PBT for more common tumours from a neurosurgical viewpoint such as meningiomas add to the competitive field of non-invasive treatments for such tumours. Until recently, PBT did not have to stand much competition from other non-invasive treatment options such as stereotactic singlesession photon-beam radiosurgery (RS) because of the rarity of the tumours treated or the paediatric age group undergoing PBT. Accordingly, PBT has been considered as part of radiation oncology, performed by radiation oncologists dealing with a rather narrow niche in terms of tumours and patients. This is going to change with the inroad of PBT into the field of more common neurosurgical neoplasms in adult patients. With the treatment of classical neurosurgical neoplasms, PBT will have to face competitive challenges and critical questions, which so far it may not have confronted. The neurosurgeon Lars Leksell developed the concept of RS and the first dedicated machine for such treatments, the Gamma Knife (GK). The neurosurgeon

Thomas Mindermann

tmindermann@hin.ch

1 Neurosurgery, Klinik Im Park, Seestrasse 220, 8027 Zurich, Switzerland

2 Medical Physics, Klinik Hirslanden, Zurich, Switzerland
John R. Adler developed the second dedicated machine for $\mathrm{RS}$, the CyberKnife (CK). RS was introduced by neurosurgeons against the resistance of radiation oncologists. Because of that resistance and in advocacy of their new concept, neurosurgeons amassed an unparalleled knowledge and experience in RS for intracranial neoplasms. As a consequence, RS is nowadays one of the best-documented medical treatments, with more than 15,000 publications on Gamma Knife radiosurgery (GKRS) alone. Ideally, neurosurgeons approach tumours such as intracranial meningiomas with a concept in mind including microsurgery, RS or the combination of both. Therefore, any new treatment for classical neurosurgical neoplasms of adult patients will have to prove at least equality of results with well-established neurosurgical treatment options such as RS, stand the test of everyday clinical practice and compete with the comparably low costs of RS.

Until today, the "gold standard" for any radiation treatment of WHO grade I meningiomas remains GKRS. Altogether, more than 100,000 patients with WHO grade I meningiomas have been treated with single-session GKRS worldwide and multicentre studies with up to 5,300 benign meningiomas in one study alone have been published, revealing excellent long-term results [2]. In addition, studies on GKRS for meningiomas exist for specific anatomical locations such as cavernous sinus, parasagittal location, skull base, foramen magnum, etc., and for specific technical aspects such as volume-staging, conformality index as a predictor to perifocal oedema formation, etc. This is an unparalleled knowledge base from which neurosurgeons may draw in planning their RS treatments. Over the years, the excellent results and the broad acceptance of RS for meningiomas has led to modifications in the surgical treatment of meningiomas. Often, a radical resection of a larger tumour or a smaller tumour in challenging locations may be avoided by leaving a tumour remnant for radiosurgery and thereby reducing the patients' risks. Today, many meningiomas 
are treated upfront by RS, depending on their size and location. Meningiomas in delicate locations especially, such as the cavernous sinus, respond well to RS, either restoring the patients' neurological function or leaving it intact. It goes without saying that a neurosurgeon's anatomical knowledge of the brain and the specific condition to be treated contributes to this extraordinary success. Ideally, the treating neurosurgeon or radiosurgeon has been part of the surgical team when tumour remnants are to be treated or has enough surgical routine so that all the surgical and anatomical aspects of postoperative images are well understood. This is especially important when it comes to skull base tumours or pituitary adenomas. Any alternative radiation technique faces stiff competition for all of those reasons. PBT will have to compare and compete with the excellent results of RS not only in the treatment of meningiomas but also in the treatment of other standard neurosurgical neoplasms such as vestibular schwannomas, pituitary adenomas or cerebral metastases. So far, only a limited number papers on various and non-standardised forms of PBT for WHO grade I meningiomas have been published in English, covering only a few hundred patients, including this issue's study. In some of those papers, patients had not only benign but also higher-grade meningiomas and some underwent additional external photon-beam radiation, thus reducing the knowledge base on PBT even further.

In this context, several technical and clinical issues need to be addressed by centres delivering PBT, such as target motion, proton beam delivery, target conformality, the range of suitable tumour volumes, protection of organs at risk (OAR), adequate dose, hypofractionation and many more. Over the past 3 decades, basically all of those issues have been addressed and optimised in RS with dedicated devices such as the GK or the CK. This lead of RS is not easy to be on a par with. An incomplete compilation of the issues addressed successfully by RS include challenges with image acquisition, distortion correction, target motion, tumour size, dose escalation, target conformality, collimator size for a steepest possible dose fall-off, dose maximum at OAR, single-session treatment versus hypofractionation, dose-staging versus volume-staging, and many more. An added margin of $5 \mathrm{~mm}$ to the target volume as in the authors' paper or any other added margin such as 2 or $3 \mathrm{~mm}$ as in other publications would be unthinkable in RS. It would just mean that the precision of target location and beam delivery is insufficient for single-session treatment. Other issues specific to PBT, such as the method of proton beam delivery (scattering vs spot-scanning), which influences target conformality, need to be addressed. It seems that spot-scanning with the superposition of so-called spots leads to a better target conformality than scattering. Under best current clinical conditions, a target volume of $1-6 \mathrm{~cm}^{3}$ will be hit by about $10-60$ spots delivered by a gantry with two arrival angles. In comparison, GKRS typically treats a target volume of 1-6 $\mathrm{cm}^{3}$ with about 12-24 isocentres or "spots", which are delivered from more than 2,000 arrival angles, from 201 entry points, distributed evenly over a hemispheric helmet covering the patient's head. This spherical geometric arrangement of entry points around the head results in a far better target conformality and, accordingly, in a superior protection of OAR. The full or partial inclusion of parts of the optic apparatus in the radiation field in $66 \%$ of patients, as reported in this issue's publication on PBT [3], would be unacceptable in RS. Target conformality of PBT may be sufficient for other organs, such as prostate or pancreas, but it is not for the brain. In that sense, PBT is still a developing technique when it comes to the treatment of benign intracranial meningiomas. Hopefully, PBT will adopt some of the technical finesse of RS, with multiple entry points and arrival angles. Until that happens, results will inevitably be inferior to RS concerning target conformality, protection of OAR and clinical outcome.

According to the literature, complications associated with PBT are: radiation induced optic neuropathy (RION), acute demyelinisation, lethal focal radiation necrosis, expansive tumour cysts, large vessel cerebral vasculopathy, pituitary insufficiency, hearing loss, memory deficits, neutron-induced secondary cancers, etc. Most of those complications can only be ascribed to excessive dose outside the target. Evidently, PBT's theoretical physical advantages of the Bragg peak phenomenon and the $10 \%$ higher relative biological effectiveness do not necessarily translate into a clinical advantage over RS, mostly due to geometrical disadvantages. RION and the other complications, some of which are specific to PBT, should never be seen in either RS or PBT. One may speculate that in the future the delivery of other heavy particles such as carbon ions with a higher ionisation density than protons may enable more geometrical degrees of freedom and therefore lead to superior results. PBT would then serve as a bridging technology.

It is of great value to see this study's [3] results of PBT in patients with WHO grade I meningiomas. Unfortunately, they do not compare convincingly to results of RS for the same tumour class. This may have to do with patient selection, tumour size, specific technical properties inherent to PBT or any combination of the above. The authors' paper raises a number of issues. Is it really reasonable to use PBT for tumour volumes up to $64 \mathrm{~cm}^{3}$ or an average tumour volume of $13 \mathrm{~cm}^{3}$ ? If PBT should be considered for larger tumours, why not adopt radiosurgical techniques such as volume- or dose-staging? Is hypofractionation for benign tumours really the answer to problems with target conformality? Why not combine microsurgery with PBT in order to reduce target volumes? Many questions remain unanswered and PBT may still profit a lot from adopting neurosurgical approaches to classical neurosurgical neoplasms. Therefore, the title's claim that "hypofractionated high-energy proton beam irradiation is an alternative treatment for WHO grade I meningiomas" [3] needs to be questioned. Is that really so? In view of the published data, the small patient numbers, the non-uniform delivery mode in those publications, the issues raised concerning 
PBT and, last but not least, the excellent alternative treatment option of RS, PBT cannot be classified as an established treatment option for benign intracranial meningiomas and should be identified as such to patients and caregivers alike.

\section{References}

1. Levin WP, Kooy H, Loeffler JS, TF DL (2005) Proton beam therapy. Br J Cancer 93:849-854

2. Santacroce A, Walier M, Régis J, Liscak R, Motti E, Lindquist C, Kemeny A, Kitz K, Lippitz B, Alvarez RM, Pedersen P-H, Yomo S,
Lupidi F, Dominikus K, Blackburn P, Mindermann T, Bundschuh O, van Eck ATCJ, Fimmers R, Horstmann GA (2012) Long-term tumor control of benign intracranial meningiomas after radiosurgery in a series of 4565 patients. Neurosurgery 70:32-39

3. Vlachogiannis P, Gudjonsson O, Montelius A, Grusell E, Isaacsson U, Nilsson K, Blomquist E (2017) Hypofractionated high-energy proton beam irradiation is an alternative treatment for WHO grade I meningiomas. Acta Neurochir. https://doi.org/10.1007/s00701017-3352-4

4. Waddle MR, Sio TT, Van Houten HK, Foote RL, Keole SR, Schild SE, Laack N, Daniels TB, Crown W, Shah ND, Miller $\mathrm{RC}$ (2017) Photon and proton radiation therapy utilization in a population of more than 100 million commercially insured patients. Int J Radiat Oncol Biol Phys. https://doi.org/10.1016/j. ijrobp.2017.07.042 